\title{
Acceptance of Teleconsultation Among Doctors and The Determinant Factors in Teaching Hospital in Malaysia
}

\author{
Laila Abushaar \\ Department of Community Health Sciences, Faculty of \\ Medicine \\ Universiti Kebangsaan Malaysia \\ Cheras, Malaysia \\ saleh_laila@yahoo.com
}

\author{
Aniza Ismail ${ }^{2}$ \\ Department of Community Health Sciences, Faculty of \\ Medicine \\ Universiti Kebangsaan Malaysia \\ Cheras, Malaysia \\ aniza@ppukm.ukm.edu.my
}

\begin{abstract}
The recent introduction of information technology has improved the quality of healthcare services. In many developing countries, including Malaysia, patients suffer from a shortage of trained medical specialists and Tele-consultation would ease the access to the specialists. We need to know if doctors are willing to accept such technology since they are the end users. The objective of the study is to measure the acceptance of Tele-consultation among doctors in Hospital of Conselor Tuanku Muhriz (HCTM) and to identify the factors which influence the acceptance of Teleconsultation among them such as Socio-demographic factor, knowledge, attitude, perceived ease of use and perceived usefulness towards Tele-consultation. Those factors were chosen according to the technology acceptance model (TAM) and some literature. This is a cross sectional study based on a quantitative method. Data was collected from HCTM outpatient clinics in Cheras area, Kuala Lumpur in March 2017. A total of 90 questionnaires were collected from eight different outpatient clinics in HCTM. SPSS (version 22.0) was used to analyse the data. The results showed acceptance rate of $63.3 \%$ among doctors in UKMM. The study also showed that perception of usefulness and the positive attitude had a significant association with the acceptance of $\mathrm{TC}$. In conclusion, this study has revealed the importance of factors that have influenced Tele-consultation acceptance as perceived usefulness and positive attitude. Furthermore, the overall findings from the study suggest that TAM is an appropriate model for explaining physicians' technology acceptance decisions.
\end{abstract}

Keywords- Telemedicine; Perception; Tele-consultation; Acceptance; Doctors

\section{INTRODUCTION}

Technological discoveries are reforming the way of delivering healthcare services to the public. Modern technology has altered the construction and organization of the entire medical field. Starting from the universal embracing of electronic medical records to the progress in biomedical engineering and technology, modern healthcare and its provision is shifting dramatically. Malaysia is one the countries that made many amendments in advance of health information implementation into the health system. The concept of the term "Paperless hospitals" started in 1996-2000 through the seventh Malaysian plan by introducing a fully computerized system for electronic medical records, telemedicine, and teleconferencing [1]. Improving Health Information Management was one of the primary intentions to attain the vision of the 9th Malaysian plan to ensure the Integration of Health information Management into all healthcare organizations of the country [2]. Tele-consultation is one form of Telemedicine that does not use a direct 'face to face' or 'in person' consultation in order to diagnose or followup any medical condition. In many developing countries, including Malaysia, patients usually face lack of trained medical specialists especially in rural areas[3]. Many patients are forced to wait for a while before they get to see a specialist. In many cases, general practitioners are forced to refer patients with complicated medical cases from their primary health centre to a tertiary centre due to lack of available specialists.

The objectives of this study is To assess the acceptance of TC among doctors at outpatient clinics in tertiary teaching hospitals and to identify the factors that influence the acceptance of Tele-consultation among doctors in Hospital of Conselor Tuanku Muhriz (HCTM) including Sciodemographic (Age, sex, education level, medical specialty, years of experience, and computer literacy), Knowledge, attitude, perceived ease of use and perceived usefulness towards Tele-consultation. This study explored user technology acceptance in healthcare organization planning to provide telemedicine care and services soon. Tele-consultation could be either uses the store-and-forward method (asynchronous) which, patient medical information is compressed in digital matter, and sent via the network such as e-mail to a known specialist or physician. Asynchronous is found to be feasible for developing and developed countries as well since it provides a low-cost telemedicine for clinical purposes $[4,5]$. Another method to use TC is in an interactive way (synchronous), which involves the use of video conferencing between the sender and the receiver for the purpose of medical advice. Such method is less implemented in developing countries compared to the synchronous mode due to the inappropriate telecommunication infrastructure in these countries[5].

Telehealth was implemented by the Malaysian government under the Multimedia Super Corridor (MSC) project, Telehealth Flagship in 1996. This project was one of the seven flagship applications of MSC grouped under the 'Multimedia Development Flagship Application' and has great knowledge with reasonable depth on a particular topic. At first, the support provided was to access the sources of information by 
healthcare professionals via the internet to medical journals, ebooks, and medical databases and to use the e-mail and related technologies to create virtual environment for the healthcare professionals to interact and communicate electronically [3, 6]. The Telemedicine Act 1997 endorsed the growth of information communication technology (ICT) in the health sector. The Ministry of Health wanted to manage patient information by linking systems and providers through a solid program of information communication technology start out the telemedicine plan which included the Multimedia Super Corridor Flagship initiatives, Program/function-based enterprises include the Nationwide Health Management Information System, State-wide Teleprimary Care, and Public/Client Access

\section{Technology Acceptance Model Theory}

First, Technology Acceptance Module (TAM) which is a global module that was proposed by Davis in 1989 used to evaluate the acceptance of any new technology[7]. This module processes the behavior- related part of the attitude in order to differentiate between belief and attitude towards the new technology by setting up relation of the two factors (perceived ease of use and perceived usefulness). Such acceptance is appraised by how users' perceived ease of use, perceived usefulness and the attitude towards any new technology. Perceived ease of use (PEOU) evaluated by appraising how much users believe that the new technology is free of any mental and physical effort. On the other hand, perceived usefulness (PU) is evaluated by appraising how much the user believes that using a particular technology will enhance the diagnosis and treatment of patients thus improving health care practices $[8,9]$.

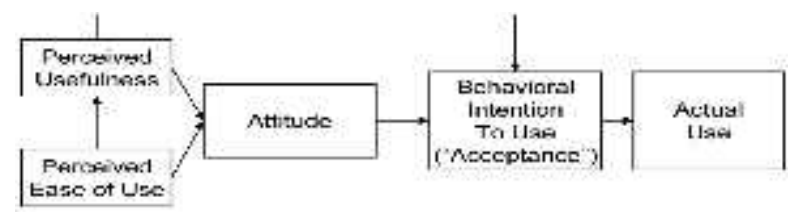

Figure 1: Technology Acceptance Model

\section{METHODOLOGY}

This study is using a quantitative survey arranged by a definite questionnaire. In this study, a descriptive study using a cross-sectional survey was established to assess associations between the variables by accumulating data from The Hospital of Conselor Tuanku Muhriz (HCTM), a teaching hospital in Cheras, Malaysia. The duration of this study was From February to June 2017 while doctors in outpatient clinics in HCTM where chosen. The data for this correlational study were gathered utilizing a survey questionnaire administered to medical officers and specialists who practice medicine in the outpatient department of HCTM. HCTM center was chosen because it is not only a tertiary centre but also a teaching hospital and a research institution; where intranet solution for teleconferencing is being implemented for educational purposes and that Tele-consultation could be easier carried out in the near future. The departments involved were internal medicine, surgery, orthopedic, gynecology and obstetrics, pediatrics, ENT, hematology, and family medicine departments and list of physicians from the Human Resources department with total 117 names. The choice of doctors from the HCTM was based on the proportion of doctors at that department and the Likelihood of their participation in the study.

Doctors were given a 4-page paper questionnaire regarding Tele-consultation asking about six main sections (Sociodemographic, Knowledge, PEOU, PU, Attitude towards TC, Acceptance of TC) The design of validated questionnaires regarding all sections of the questionnaire except knowledge section were adapted and modified from two similar studies $[3,10,11]$. The researcher developed the other set of questionnaires regarding the knowledge section according to the objectives of the study and derived from related literature reviews. Although validated by prior research, the adopted instrument was examined to ensure content validity and reliability within the targeted context by the judgment of a panel of experts including two experts in health technology, three doctors of public health, and three physicians from altered medical specialty/subspecialty to weigh content validity and to confirm the relevancy of the questions to the objectives of the study. A pre-test was done among 26 doctors inside and outside HCTM who were excluded on real study and Reliability was tested by using the Cronbach's Alpha in SPSS, and all sections of the questionnaire gave a result of 0.882 that represents a commonly acceptable level for exploratory research.

Data was analyzed using SPSS version 22. Descriptive analysis was carried out by calculating frequencies and percentages. Association was identified between independent and dependent variables using Chi-square. After running initial frequencies, the bivariate was carried out using chi square and multivariate analysis was carried out using regression analysis for linking association between factors and outcome. Proposal got the approval from the ethical committee of UKM, the Dean's office and head of outpatient departments were taken before proceeding to data collection.

\section{RESULTS}

Out of 110 questionnaires distributed, 90 were completed and returned which represents $83 \%$ response rate. The majority of the participants were aged below 40 of years $(91.1 \%)$. Male gender represented half of the respondents (51.1\% vs $48.9 \%$ female). The highest numbers of participants $(25.6 \%)$ were from Gynaecology and Obstetrics department followed by the orthopaedic and internal medicine department $(22.2 \% \& 20 \%)$ then other departments were followed. The majority of participants $(75.6 \%)$ obtained MBBS degree as their highest level of education, while only $15.6 \%$ obtained Master Degree and 8.9\% obtained other certificates after their MBBS. No participant had PhD degree in this sample. Around $71 \%$ of participants have between 5-10 years of experience in health sector while $19 \%$ have more than 10 years of experience and only $10 \%$ of participants have experience between 1-5 years 
After running the Chi square analysis of the data (Table 1) we found association between Perception of usefulness and acceptance of TC, $\mathrm{X} 2=10.421, \mathrm{~N}=90$; d.f. $=1 ; \mathrm{p}<0.05$. 39. Phi and Cramer's V value of 0.34 shows moderate strength of association. There was an association found in the attitude towards Tele-consultation with acceptance of TC, X $2=$
11.553, $\mathrm{N}=90 ;$ d.f. $=1 ; \mathrm{p}<0.05$. Phi and Cramer's V value of 0.35 shows moderate strength of association. Other factors such as socio-demographic, Knowledge and Perceived ease of use did not show association with the acceptance of TC among doctors in HCTM

TABLE 1. ASSOCIATION BETWEEN TC ACCEPTANCE WITH OTHER FACTORS

\begin{tabular}{|c|c|c|c|c|c|c|}
\hline \multirow{2}{*}{ Variable } & \multicolumn{2}{|c|}{ Acceptance of TC } & \multicolumn{2}{|c|}{ No Acceptance } & \multirow{2}{*}{$x^{2}$} & \multirow{2}{*}{ P-value } \\
\hline & $n$ & $\%$ & $n$ & $\%$ & & \\
\hline Age & & & & & \multirow{5}{*}{3.823} & \multirow{5}{*}{0.281} \\
\hline 30 to 39 & 32 & 39 & 50 & 61 & & \\
\hline 40 to 49 & 0 & 0 & 5 & 100 & & \\
\hline 50 to 59 & 1 & 50 & 1 & 50 & & \\
\hline 60 and above & 0 & 0 & 1 & 100 & & \\
\hline \multicolumn{5}{|l|}{ Sex } & \multirow{3}{*}{.667} & \multirow{3}{*}{.414} \\
\hline Male & 15 & 32.6 & 31 & 67.4 & & \\
\hline Female & 18 & 40.9 & 26 & 59.1 & & \\
\hline \multicolumn{5}{|l|}{ Medical Speciality } & \multirow{9}{*}{5.332} & \multirow{9}{*}{.620} \\
\hline Hematology & 0 & 0 & 1 & 100 & & \\
\hline Family Medicine & 1 & 33.3 & 2 & 66.7 & & \\
\hline ENT & 0 & 0 & 5 & 100 & & \\
\hline General Surgery & 5 & 33.3 & 66.7 & 100 & & \\
\hline Orthopedic & 8 & 40 & 12 & 60 & & \\
\hline Obs \& Gyn & 8 & 34.8 & 15 & 65.2 & & \\
\hline Internal Medicine & 8 & 44.4 & 10 & 55.6 & & \\
\hline paediatrics & 3 & 60 & 2 & 40 & & \\
\hline \multicolumn{5}{|l|}{ Highest grade obtained } & \multirow{4}{*}{3.699} & \multirow{4}{*}{.157} \\
\hline MBBS & 25 & 36.8 & 43 & 63.2 & & \\
\hline Master & 3 & 21.4 & 11 & 78.6 & & \\
\hline Others & 5 & 62.5 & 3 & 37.5 & & \\
\hline \multicolumn{5}{|c|}{ years of service in health sector } & \multirow{4}{*}{.528} & \multirow{4}{*}{.768} \\
\hline 1-5 Years & 4 & 44.4 & 5 & 55.6 & & \\
\hline 5-10 Years & 22 & 34.4 & 42 & 65.6 & & \\
\hline More than 10 years & 7 & 41.2 & 10 & 58.8 & & \\
\hline \multicolumn{5}{|c|}{ Feel comfortable with technology } & \multirow{3}{*}{2.649} & \multirow{3}{*}{.104} \\
\hline yes & 30 & 34.9 & 56 & 65.1 & & \\
\hline No & 3 & 75 & 1 & 25 & & \\
\hline \multicolumn{5}{|l|}{ Knowledge } & \multirow{2}{*}{1.420} & \multirow{2}{*}{.233} \\
\hline Poor knowledge & 16 & 48.5 & 17 & 51.5 & & \\
\hline
\end{tabular}




\begin{tabular}{|c|c|c|c|c|c|c|}
\hline Good knowledge & 17 & 29.8 & 40 & 70.2 & & \\
\hline PEOU & & & & & \multirow{3}{*}{1.657} & \multirow{3}{*}{.198} \\
\hline No PEOU & 11 & 47.8 & 12 & 52.2 & & \\
\hline PEOU & 22 & 32.8 & 45 & 67.2 & & \\
\hline PU & & & & & \multirow{3}{*}{10.421} & \multirow{3}{*}{.001} \\
\hline No PU & 22 & 55 & 18 & 45 & & \\
\hline PU & 11 & 22 & 39 & 78 & & \\
\hline \multicolumn{5}{|l|}{ Attitude } & \multirow{3}{*}{11.553} & \multirow{3}{*}{.001} \\
\hline Negative attitude & 22 & 56.4 & 17 & 43.6 & & \\
\hline Positive attitude & 11 & 21.6 & 40 & 78.4 & & \\
\hline
\end{tabular}

As showed in (Table 2) only two independent variables made a unique statistically significant contribution to the model (perceived Usefulness and positive attitude) when Simple Logistic Regression was done while only positive attitude was statistically significant when Multiple Logistic Regression was done. This study showed that doctors who are accepting TC were over 4 times more likely to have positive attitude towards
TC than who had negative attitude towards TC. Similarly, doctors who are accepting TC were over 4 times more likely to have perception of usefulness of TC compared to doctors who had no perception of usefulness of TC with p-value < 0.05 , after controlling of all other factors in the model, yet this factor was no longer a significant predictor for TC when Multi Logistic Regression was done.

TABLE II. FINAL MODEL FOR FACTORS ASSOCIATED WITH TC USING LOGISTIC REGRESSION

\begin{tabular}{|c|c|c|c|c|c|c|}
\hline & \multicolumn{3}{|c|}{ Simple logistic regression } & \multicolumn{3}{|c|}{ Multiple logistic regression } \\
\hline & B & Crude OR (95\% CI) & $\mathbf{p}$ & B & Adjusted OR (95\% CI) & $\mathbf{p}$ \\
\hline Sex(Male) & 0.358 & $1.431(.605-3.384)$ & .415 & 0.879 & $2.401(0.605-9.596)$ & 0.213 \\
\hline Age & -0.446 & $0.640(0.39-10.60)$ & .755 & 37.425 & $1.792(0.00-\ldots)$ & 0.999 \\
\hline Medical specialty & -0.264 & $0.768(0.566-1.041)$ & 0.089 & 20.197 & $590754(0.00-\ldots)$ & 1.00 \\
\hline Years of service & -0.11 & $0.989(0.440-2.220)$ & 0.978 & 0.057 & $1.059(0.175-6.395)$ & 0.950 \\
\hline Comfort with tech & 1.723 & $5.6(0.558-56.203)$ & 0.143 & 0.616 & $1.852(0.143-24.06)$ & 0.638 \\
\hline Knowledge (good) & 0.795 & $2.215(0.911-5.380)$ & 0.079 & 0.475 & $1.608(.497-5.200)$ & 0.428 \\
\hline PEOU & 0.629 & $1.875(0.715-4.917)$ & 0.201 & 0.233 & $1.263(0.347-4.595)$ & 0.723 \\
\hline PU & 1.466 & $4.333(1.737-10.811)$ & 0.002 & 1.123 & $3.073(0.839-11.253)$ & 0.090 \\
\hline Attitude(Positive) & 1.549 & $4.706(1.876-11.805)$ & 0.001 & 1.479 & $4.389(1.162-16.578)$ & 0.029 \\
\hline \multicolumn{7}{|c|}{$\begin{array}{l}\text { Adjusted r2: } 0.55 \\
\text { Direct logistic regression method applied. } \\
\text { Multicollinearity and interaction term were checked and not found (Tolerance }>0.1) \\
\text { Homer }- \text { Lemeshow test }(p=0.143) \text { and Omnibus test }(\mathrm{p}=0.009) \text {, thus the fit of this model is achieved. } \\
\text { percentage accuracy in classification: PAC is } 76.7 \% \\
\text { The highlighted are the significant variables respectively }\end{array}$} \\
\hline
\end{tabular}

\section{DISCUSSION}

A total of 57 doctors had a good behavior intention to use TC when it becomes available in HCTM. This percentage is slightly lower than similar study done in Iran [12] of $75 \%$ acceptance of doctors. According to Vieru [14] doctors' higher education and experiences make them more prone to accept new technologies more quickly than students according to acceptance rate and in Italy with acceptance rate of $85 \%$ [13]. Majority of the factors studied in this paper did not have a significant influence on the acceptance. This percentage is slightly lower than what expected yet it is considered a proper outcome. There are several factors that affected the overall most TAM studies. Yet, in this study most doctors had MBBS as their highest degree $75.6 \%$ in addition TC is not yet implemented in HCTM therefore they don't have the" actual" 
experience that could affect their acceptance.Another reason affected their acceptance is the refusal of the concept of seeing patients online only even for simple illnesses. $39 \%$ disagreement rate regarding seeing patients online only for the purpose of diagnosing or prescribing medication of diseases that don't require much investigation. This phenomena is explained due to the usage of Tele-consultation in Malaysia only between doctors for referral or for second opinion therefore they are not yet adopted to the idea of seeing patients through TC as well.

Unlike Vankatesh and Au studies [15, 16] men were not more influenced by their perception of usefulness comparing to females nor females were more influenced by ease of usefulness compared to male. In terms of age the majority of participants $91.1 \%$ were between $30-39$ years of age, yet $39 \%$ of this age group did not accept TC comparing to $100 \%$ acceptance among the 40-49 age group, nor influenced their perception of usefulness unlike Au's study[16] that showed a direct relationship between perceived usefulness and behavioural intention while perceived ease of use. Study results also showed that years of experience has no significant association with the acceptance of TC unlike the literature review supported by Marrop and Win that related the experience with the training and education gain that usually affect the appreciation of TM benefits and capabilities[5].

According to $\mathrm{Au}$ and Ayatollahi [12, 16] knowledge is considered an essential influence of perception and attitude toward telemedicine. The overall knowledge level is considered appropriate $[63.3 \%)$ of answering at least 8 questions out of 10 comparing to similar study in Iran of $91 \%$ of poor knowledge level among doctors [12]. This is due to their previous knowledge on the concept of telemedicine and tele-consultation $(57.8 \%)$ although they don't use them in HCTM.

The results of this study was similar to the outcome of a Chau and Hu's study [17] when they stated that physicians have the relatively high general competence and mental/cognitive capacity and may comprehend the use of technology quickly; that is, become familiar with its operations without going through the intense training that might be necessary among other user populations. Alkmim [18] also indicated that the effect PEOU varies with different authors and studies yet $93 \%$ of the participants in his study exhibited PEOU compared to only $67 \%$ in our findings. Furthermore, physicians in many cases have relatively high staff support for operating medical equipment and related technologies. Together, these factors might have contributed to physicians' placing less weight on perceived ease of use

The overall PU among physicians was only $55.6 \%$ which is considered lower than parallel study that exhibited Perception of usefulness of $71 \%$ up to $95 \%$ [13] and a $86 \%$ perception of usefulness in Italy [19]. Yet, majority of respondents who had perception of usefulness of TC actually accepted TC although many some doctors did not perceive the usefulness of TC in following up of chronic diseases like DM, yet that did not affect the overall perception of usefulness among doctors. Furthermore, there was a significant influence of PU on the acceptance. Such results comes along with Chau and $\mathrm{Hu}$ statement that perceived usefulness is the most significant factor affecting physicians' acceptance of telemedicine technology. The effect of perceived usefulness on both attitude and behavioural intention were consistently the highest among all other models examined in their study. Judging by the direct and indirect effect (via attitude) on behaviour intention, perceived usefulness is likely to insert significant influences on a physicians' intention to use telemedicine technology[17]. Yet, this factor was excluded of being a determinant due to the insignificant results for both direct and backwards Multiple Logistic Regression analysis were done

Both bivariate and multivariate analysis showed significant influence of positive attitude on the acceptance. Such results comes along with $\mathrm{Chau}$ and $\mathrm{Hu}$ statement about the critical role of attitude in technology acceptance decision making by individual professionals and therefore singles out the importance of attitude cultivation and management to successful technology implementation[17]. Only $56.7 \%$ of doctors showed positive attitude towards TC and such outcome was due the majority concerns $(78.9 \%)$ about the loss of effective communications between doctors and patient, in addition (87.8\%) of respondents showed concerns about patient privacy/confidentiality and $81.1 \%$ showed their concerns about the high cost of the equipment used in TC implementation. Yet, such concerns did not affect the overall influence of attitude on the acceptance of TC among doctors. We expect that more physician would have a positive attitude towards TC after the actual usage by elimination of such concerns with the actual practice and by increasing their perception of ease of use which would affect their attitude. Such expectation is also established in another similar study where physicians' attitude has changed after execution of TC [19]

\section{CONCLUSION AND RECOMMENDATIONS}

The study has highlighted the acceptance of Teleconsultation among doctors in HCTM. It used a cross sectional study method to gather data from doctors in the outpatient department of HCTM. . Acceptance rate among doctors in HCTM was moderate which was lower than similar studies. Majority of the factors studied in this paper did not have a significant influence on the acceptance except perception of usefulness and positive attitude. Such lower acceptance rate might due to lack of "actual" experience of TC or due to having MBBS as the highest degree obtained among participants. Male gender, years of experience, technology literacy and medical specialty did not influence the acceptance of TC unlike similar studies [5]. In addition good knowledge and perception of ease of use did not have influence on the acceptance of TC unlike similar studies $[12,17]$.

The study has significant impact on technology adoption studies in health care in Malaysia. The study has revealed the importance of factors that have influenced Tele-consultation acceptance which have established a significant role in the acceptance of the technology in health care environment. These aspects were found to be Perceived usefulness of TC and positive attitude towards TC. In addition, the overall, findings from the study suggest TAM be an appropriate model 
for explaining individual physicians' technology acceptance decisions.

It is recommended that doctors get proper training before implementing Tele-consultation in HCTM so they gain more perception about the usefulness of TC and more perception of ease of use such technology. Proper training also would widen their horizon about the application of diagnostic and management methods when it comes to distant telecommunication with patients and not only with doctors. It is also recommended that the government along with stakeholders arrange the legal framework for any rules and regulations related to telemedicine and deal with the telemedicine issues, such as confidentiality, reliability, liability and cross-border authority and Providing the equipment required for implementation of telemedicine is a key factor in finding good suppliers and providers of the hardware, and the software of telemedicine required for the implementation. Such availability would help users get familiar with technology which would affect their perception and acceptance.

\section{REFERENCES}

[1] Merican I, bin Yon R. Health care reform and changes: the Malaysian experience. Asia-Pacific Journal of Public Health. 2002;14(1):17-22.

[2] Selvaraju DDS. Health information management: Malaysian experience. Health Informatics Center, Ministry of Health, Malaysia. 2006.

[3] Maarop N, Win KT. Understanding the Need of health care providers for teleconsultation and technological attributes in relation to the acceptance of teleconsultation in Malaysia: A mixed methods study. Journal of medical systems. 2012;36(5):2881-92.

[4] Yusof K, Neoh KHB, bin Hashim MA, Ibrahim I. Role of teleconsultation in moving the healthcare system forward. AsiaPacific Journal of Public Health. 2002;14(1):29-34.

[5] Maarop N. Understanding the acceptance of teleconsultation technology in Malaysian government hospitals. 2013.

[6] Som MM, Norali A, Ali MM, editors. Telehealth in Malaysia-An overview. Industrial Electronics \& Applications (ISIEA), 2010 IEEE Symposium on; 2010: IEEE.

[7] Mascha MF, Adya M. USING THE TECHNOLOGY ACCEPTANCE MODEL IN PREDICITNG ACCEPTANCE OF IMPOSED TECHNOLOGY: A FIELD STUDY. Marquette University. 2010.

[8] Abu-Dalbouh HM. A questionnaire approach based on the technology acceptance model for mobile tracking on patient progress applications. Journal of Computer Science. 2013;9(6):76370.

[9] Kifle M, Mbarika VW, Datta P. Telemedicine in sub Saharan Africa: The case of teleophthalmology and eye care in Ethiopia. Journal of the American Society for Information Science and Technology. 2006;57(10):1383-93.

[10] Gagnon MP, Orruno E, Asua J, Abdeljelil AB, Emparanza J. Using a modified technology acceptance model to evaluate healthcare professionals' adoption of a new telemonitoring system. Telemedicine and e-Health. 2012;18(1):54-9.

[11] D.W.Green. The Telemedicine Project 1999 [Available from: http://www.dr-green.co.uk/html/telemed_project.htm.

[12] Ayatollahi H, Sarabi FZP, Langarizadeh M. Clinicians' Knowledge and Perception of Telemedicine Technology. Perspectives in Health Information Management. 2015.

[13] Zanaboni P, Scalvini S, Bernocchi P, Borghi G, Tridico C, Masella C. Teleconsultation service to improve healthcare in rural areas: acceptance, organizational impact and appropriateness. BMC Health Services Research. 2009;9(1):238.
[14] Vieru D. A model for telemedicine adoption: A survey of physicians in the provinces of Quebec and Nova Scotia: Concordia University; 2000.

[15] Venkatesh V, Morris MG. Why don't men ever stop to ask for directions? Gender, social influence, and their role in technology acceptance and usage behavior. MIS quarterly. 2000:115-39.

[16] Au L. Assessing the potential needs for Telehealth in Papua New Guinea (PNG). 2009.

[17] Chau PY, Hu PJ-H. Investigating healthcare professionals' decisions to accept telemedicine technology: an empirical test of competing theories. Information \& management. 2002;39(4):297311.

[18] Alkmim MB, Marcolino MS, Figueira RM, Sousa L, Nunes MS, Cardoso CS, et al. Factors associated with the use of a teleconsultation system in Brazilian primary care. Telemedicine and e-Health. 2015;21(6):473-83.

[19] B L, E A, F B, F D, C E, E G, et al. Analysis of user-satisfaction with the use of a teleconsultation system in oncology. Medical Informatics and the Internet in Medicine. 2003;28(2):73-84. 